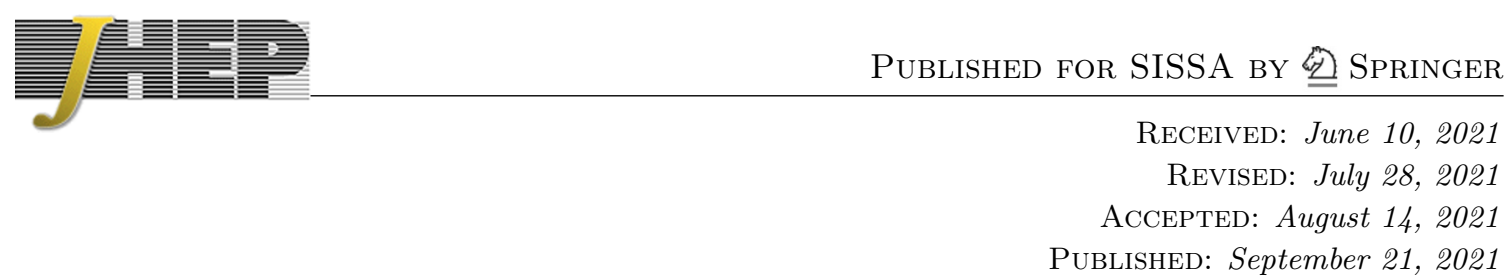

\title{
Beautiful and charming chromodipole moments
}

\author{
Ulrich Haisch and Gabriël Koole \\ Max Planck Institute for Physics, \\ Föhringer Ring 6, 80805 München, Germany \\ E-mail: haisch@mpp.mpg.de, koole@mpp.mpg.de
}

ABstract: In the context of the Standard Model effective field theory we derive direct and indirect bounds on chromodipole operators involving the bottom and charm quark. We find that the experimental upper limit on the neutron electric dipole moment puts severe constraints on the imaginary parts of the Wilson coefficients of both chromodipole operators. The magnitudes of the Wilson coefficients are instead only weakly constrained by dijet searches and $Z$-boson production in association with bottom-quark jets. Flavour physics does not provide meaningful bounds.

Keywords: Beyond Standard Model, Effective Field Theories

ArXiv EPrint: 2106.01289 


\section{Contents}

1 Introduction 1

2 Constraints from dijet angular distributions 2

3 Constraints from searches for $b$-jet pairs $\quad 6$

4 Constraints from $Z$-boson production in association with $b$-jets $\quad 8$

5 Constraints from flavour physics 10

6 Constraints from nEDM 11

$\begin{array}{lll}7 & \text { Discussion } & 14\end{array}$

\section{Introduction}

Bottom quarks play an important role in the physics of the Standard Model (SM) Higgs boson since $\mathrm{BR}(h \rightarrow b \bar{b})$ is with around $58 \%$ the largest Higgs branching ratio. Due to the large backgrounds from multi-jet production in the dominant gluon-gluon fusion Higgs production mode, the most sensitive production channels for detecting $h \rightarrow b \bar{b}$ decays are the associated production of a Higgs boson and a $W$ or $Z$ boson $(V h)$, where the leptonic decay of the vector boson enables efficient triggering. In fact, the $h \rightarrow b \bar{b}$ decay mode has been observed by both the ATLAS and the CMS collaborations at LHC Run II [1, 2], and these measurements constrain the $b \bar{b}$ signal strength in $V h$ production to be SM-like within about $25 \%$ at the level of one standard deviation.

Beyond the SM (BSM) physics modifying the bottom-Higgs dynamics is therefore only weakly constrained by existing LHC measurements with even looser limits applying in the charm-Higgs case [3-9]. In the context of the SM effective field theory (SMEFT) the dimension-six mixed-chirality operators that can lead to modifications of the $h \rightarrow b \bar{b}$ and $h \rightarrow c \bar{c}$ partial decay widths are either of Yukawa or dipole type. While the Yukawa-type operators change the rates at leading order $(\mathrm{LO})$ in $\mathrm{QCD}$, the chromodipole operators

$$
Q_{b G}=g_{s} \bar{q}_{3} \sigma_{\mu \nu} T^{a} d_{3} H G^{a, \mu \nu}, \quad Q_{c G}=g_{s} \bar{q}_{2} \sigma_{\mu \nu} T^{a} u_{2} \widetilde{H} G^{a, \mu \nu},
$$

start to contribute at the next-to-leading order (NLO) level [10]. Here $g_{s}$ denotes the QCD coupling, $T^{a}$ are the $\mathrm{SU}(3)$ colour generators and $G_{\mu \nu}^{a}$ is the gluon field strength tensor. The symbol $q_{f}$ denotes left-handed quark doublets of flavour $f$, while $u_{f}$ and $d_{f}$ are the right-handed up- and down-type quark singlets, $\sigma_{\mu \nu}=i / 2\left(\gamma_{\mu} \gamma_{\nu}-\gamma_{\nu} \gamma_{\mu}\right)$ with $\gamma_{\mu}$ the usual Dirac matrices, $H$ denotes the SM Higgs doublet and the shorthand notation $\widetilde{H}_{i}=\epsilon_{i j}\left(H_{j}\right)^{*}$ with $\epsilon_{i j}$ totally antisymmetric and $\epsilon_{12}=1$ has been used. 
In contrast to the SMEFT Yukawa-type operators that can only be constrained by processes involving a Higgs or Higgses both real or virtual, the operators (1.1) can also be bounded by measurements of observables that involve bottom (charm) quarks and possibly other particles but no Higgs boson. The goal of this work is to derive bounds on the Wilson coefficients of the operators $Q_{b G}$ and $Q_{c G}$ using existing measurements of both low- and high-energy observables of the latter type - the high-luminosity LHC (HL-LHC) constraints on (1.1) from Higgs physics can be found in the publications [11, 12] and will be studied elsewhere.

Our article is organised as follows. In section 2 we derive the present constraints from LHC Run II searches of unflavoured jet pairs, while section 3 contains a discussion of the bounds that the latest LHC searches for bottom-quark jets ( $b$-jets) provide (cf. also [12] for an earlier study). The constraints that we derive from $Z$-boson production in association with $b$-jets are discussed in section 4 . In section 5 and section 6 we present the limits from flavour physics and the neutron electric dipole moment (nEDM), respectively. We summarise our main results and give a brief discussion of the implications of our findings for explicit BSM models in section 7.

\section{Constraints from dijet angular distributions}

Jet physics has previously been used to put constraints on SMEFT operators [13-18]. In this section we will exploit searches for unflavoured jet pairs to constrain the magnitudes of the Wilson coefficients of the operators introduced in (1.1). For the case of the bottomquark chromodipole operator $Q_{b G}$, examples of contributing Feynman diagrams are shown in figure 1. The relevant collider searches look either for resonances in the dijet spectrum or analyse the angular distribution of dijet production (see [19-22] for recent LHC results).

The quantity of interest in our case is the jet angular distribution, i.e. the differential cross section for a pair of jets with invariant mass $M_{j j}$ produced at an angle $\hat{\theta}$ to the beam direction in the jet-jet centre-of-mass (CM) frame. Compared to resonance searches, the dijet angular distribution has the salient advantage that it also allows to constrain broad $s$-channel resonances or modifications in the spectrum due to the presence of higherdimensional operators in a rather model-independent fashion. This is due to the fact that the dominant channels in QCD dijet production have the familiar Rutherford scattering behaviour $d \sigma / d \cos \hat{\theta} \propto 1 / \sin ^{4}(\hat{\theta} / 2)$ at small angle $\hat{\theta}$, which is characteristic for $t$-channel exchange of a massless spin-one boson. In order to remove the Rutherford singularity, one usually considers the dijet cross sections differential in

$$
\chi=\frac{1+\cos \hat{\theta}}{1-\cos \hat{\theta}}
$$

In the small angle limit, i.e. $\chi \rightarrow \infty$, the partonic differential QCD cross section then behaves as $d \sigma / d \chi \propto$ const. Relative to the QCD background, the production of a heavy resonance or an effective operator leads to additional hard scattering and hence more jets perpendicular to the beam. In turn one expects a deviation from the QCD prediction in form of an enhanced activity of high-energetic jets in the central region of the detector. 

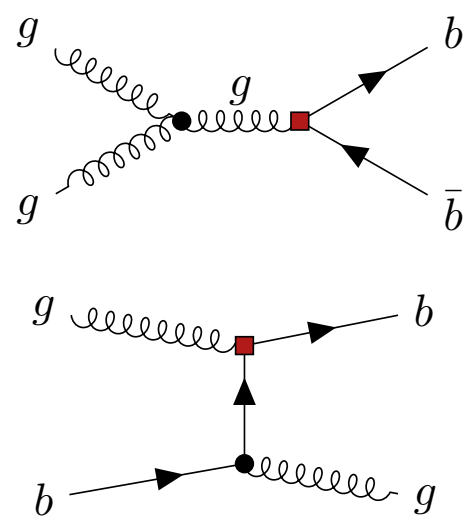
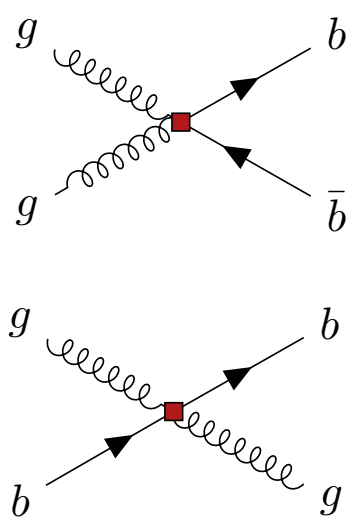

Figure 1. Tree-level diagrams leading to a correction to dijet production. The red squares denote the insertion of the bottom-quark chromomagnetic dipole moment.

If the angular distributions receive contributions from the presence of a heavy degree of freedom or an operator, one should see an excess of events in $d \sigma / d \chi$ for $\chi \rightarrow 1$ and large $M_{j j}$ with respect to the (almost) flat QCD spectrum.

By calculating the partonic differential cross section for the interference of the effective operator $Q_{b G}$ with the SM and with itself, we find the following expressions

$$
\begin{aligned}
& \left(\frac{d \sigma(g g \rightarrow b \bar{b})}{d \chi}\right)_{b G}=\frac{\pi \alpha_{s}^{2}}{2 M_{j j}^{2}}\left[\frac{4-\chi+4 \chi^{2}}{3 \chi(1+\chi)^{2}} \frac{v^{2}}{\Lambda^{2}} y_{b} \operatorname{Re}\left(C_{b G}\right)+\frac{7 M_{j j}^{2}}{3(1+\chi)^{2}} \frac{v^{2}}{\Lambda^{4}}\left|C_{b G}\right|^{2}\right], \\
& \left(\frac{d \sigma(g b \rightarrow g b)}{d \chi}\right)_{b G}=\frac{\pi \alpha_{s}^{2}}{2 M_{j j}^{2}}\left[\frac{8\left(4+9 \chi+9 \chi^{2}\right)}{9 \chi(1+\chi)^{3}} \frac{v^{2}}{\Lambda^{2}} y_{b} \operatorname{Re}\left(C_{b G}\right)+\frac{56 M_{j j}^{2}}{9(1+\chi)^{3}} \frac{v^{2}}{\Lambda^{4}}\left|C_{b G}\right|^{2}\right],
\end{aligned}
$$

where $\alpha_{s}=g_{s}^{2} /(4 \pi)$ is the strong coupling constant, $y_{b}=\sqrt{2} m_{b} / v$ is the bottom-quark Yukawa coupling with $m_{b}$ the bottom-quark mass and $v \simeq 246 \mathrm{GeV}$ the vacuum expectation value of the Higgs field. The result for the differential cross section for the process $g \bar{b} \rightarrow g \bar{b}$ is identical to the one for $g b \rightarrow g b$, and the results for the case of the operator $Q_{c G}$ are simply obtained from the above expressions by replacing $b$ everywhere by $c$. Notice that the first terms in (2.2) which arise from the interference with the SM are suppressed by one power of $y_{b}$ which provides the chirality flip needed to obtain a non-zero result. The second terms which are due to the interference of the operator $Q_{b G}$ with itself are instead enhanced by two powers of the jet-jet invariant mass $M_{j j}$. As a result the quadratic term will in practice always provide the dominant contribution to the dijet spectrum. Given that in addition the gluon-quark initiated channels are strongly suppressed by heavy-quark parton distribution functions (PDFs) this means that the limit that we derive below holds to very good approximation for both $Q_{b G}$ and $Q_{c G}$.

In terms of the partonic differential cross sections (2.2), the contribution to the double differential cross section for a pair of jets with invariant mass $M_{j j}$ produced at $\chi$ that is due to $Q_{b G}$, can be written in the following way

$$
\left(\frac{d^{2} \sigma}{d M_{j j}^{2} d \chi}\right)_{b G}=\frac{M_{j j}^{2}}{s} \sum_{\{i j\}} f f_{i j}\left(M_{j j}^{2} / s, \mu_{F}\right)\left(\frac{d \sigma(i j \rightarrow f)}{d \chi}\right)_{b G},
$$


where

$$
f_{i j}\left(\tau, \mu_{F}\right)=\frac{1}{s} \frac{2}{1+\delta_{i j}} \int_{\tau}^{1} \frac{d x}{x} f_{i / p}\left(x, \mu_{F}\right) f_{j / p}\left(\tau / x, \mu_{F}\right),
$$

are the so-called parton luminosities, the sum runs over all pairs of incoming partons $\{i j\}$ and $s$ denotes the squared CM energy of the collider. The parton luminosities are obtained from a convolution of the universal non-perturbative PDFs $f_{i / p}\left(x, \mu_{F}\right)$, which describe the probability of finding the parton $i$ in the proton with longitudinal momentum fraction $x$. The variable $\mu_{F}$ that enters (2.3) and (2.4) denotes the factorisation scale.

In figures 2 and 3 the measured normalised $\chi$ distribution for the seven different mass bins unfolded to particle level are compared to the corresponding SM predictions including both QCD and electroweak (EW) corrections at the NLO. The data and the SM predictions are both taken from the CMS analysis [19]. For comparison the normalised $\chi$ distributions assuming $v^{2} / \Lambda^{2} C_{b G}=0.5$ are also shown. The new-physics distributions are obtained at LO using CT14nnlo_as_0118 PDFs [23] with renormalisation and factorisation scale set to $M_{j j}$. These PDFs have also been used in [19] and are accessed through ManeParse [24]. The normalised $\chi$ distributions are then multiplied by a bin-wise $K$-factor to obtain a reshaped spectrum that includes effects originating from NLO QCD and EW corrections, additional non-perturbative QCD effects and the detector resolution. The $K$-factor is obtained by calculating the ratio between the central value of the CMS SM prediction and our LO SM prediction. Notice that applying the same rescaling factor to both the QCD and new-physics results is based on the assumption that the effects of the Monte Carlo (MC) shower and the event detection depend only on the invariant mass $M_{j j}$ of the dijet final state, but not on the precise form of the new-physics signal. To determine to which extend this assumption is justified would require to perform a dedicated simulation of including NLO QCD and EW corrections, parton showering and detector effects. However, such an analysis is beyond the scope of this work.

To find the $95 \%$ confidence level (CL) limit on the magnitude of the Wilson coefficient of the dipole operator $Q_{b G}$, we perform a $\chi^{2}$ fit to the normalised angular distribution in all seven dijet invariant mass bins, including both experimental and theoretical uncertainties. Minimising the $\chi^{2}$ and requiring $\Delta \chi^{2}=\chi^{2}-\chi_{\min }^{2}=3.84$ leads to

$$
\frac{\left|C_{b G}\right|}{\Lambda^{2}}<\left(\frac{1}{380 \mathrm{GeV}}\right)^{2}
$$

The same bound applies approximately also in the case of $Q_{c G}$. Notice that since the bounds (6.11) and (6.15) are significantly stronger than the latter limit, the exclusion (2.5) is in fact a bound on the real part of the Wilson coefficients $C_{b G}$ and $C_{c G}$. We add that non-zero values $v^{2} / \Lambda^{2}\left|C_{b G}\right| \simeq 0.3$ of the Wilson coefficient are, in fact, preferred by the data, and that the most sensitive bins in our $\chi^{2}$ fit are the three bins of [19] that cover the range $2.4 \mathrm{TeV}<M_{j j}<4.2 \mathrm{TeV}$ of invariant dijet masses. Notice finally that analyses of the jet-jet angular distribution in dijet production can also be used to put constraints on the chromodipole operators involving light quarks. Since the focus of this work lies on deriving constraints on the chromodipole operators involving bottom and charm quarks, we however do not entertain this possibility here. 

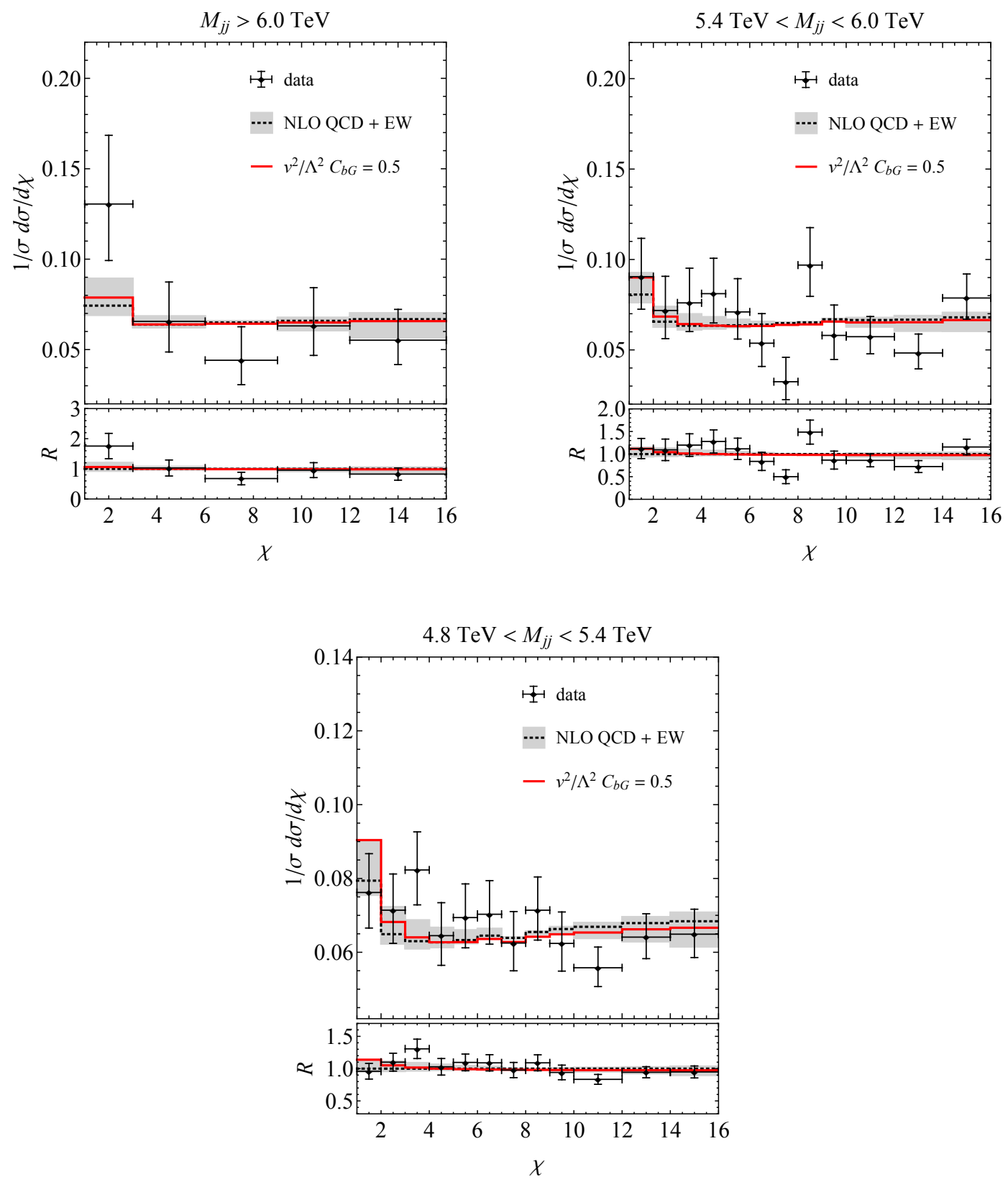

Figure 2. Normalised $\chi$ distribution in the three highest mass bins studied in the CMS analysis [19]. Unfolded data from [19] are compared to the SM prediction including NLO QCD and EW corrections (black dotted line). The error bars represent statistical and experimental systematic uncertainties combined in quadrature. Theoretical uncertainties are indicated as a grey band. Also shown is the prediction for $v^{2} / \Lambda^{2} C_{b G}=0.5$ (red line). The lower panels show the ratio of the unfolded data distributions and the NLO predictions as well as the new-physics distributions. See main text for more details. 

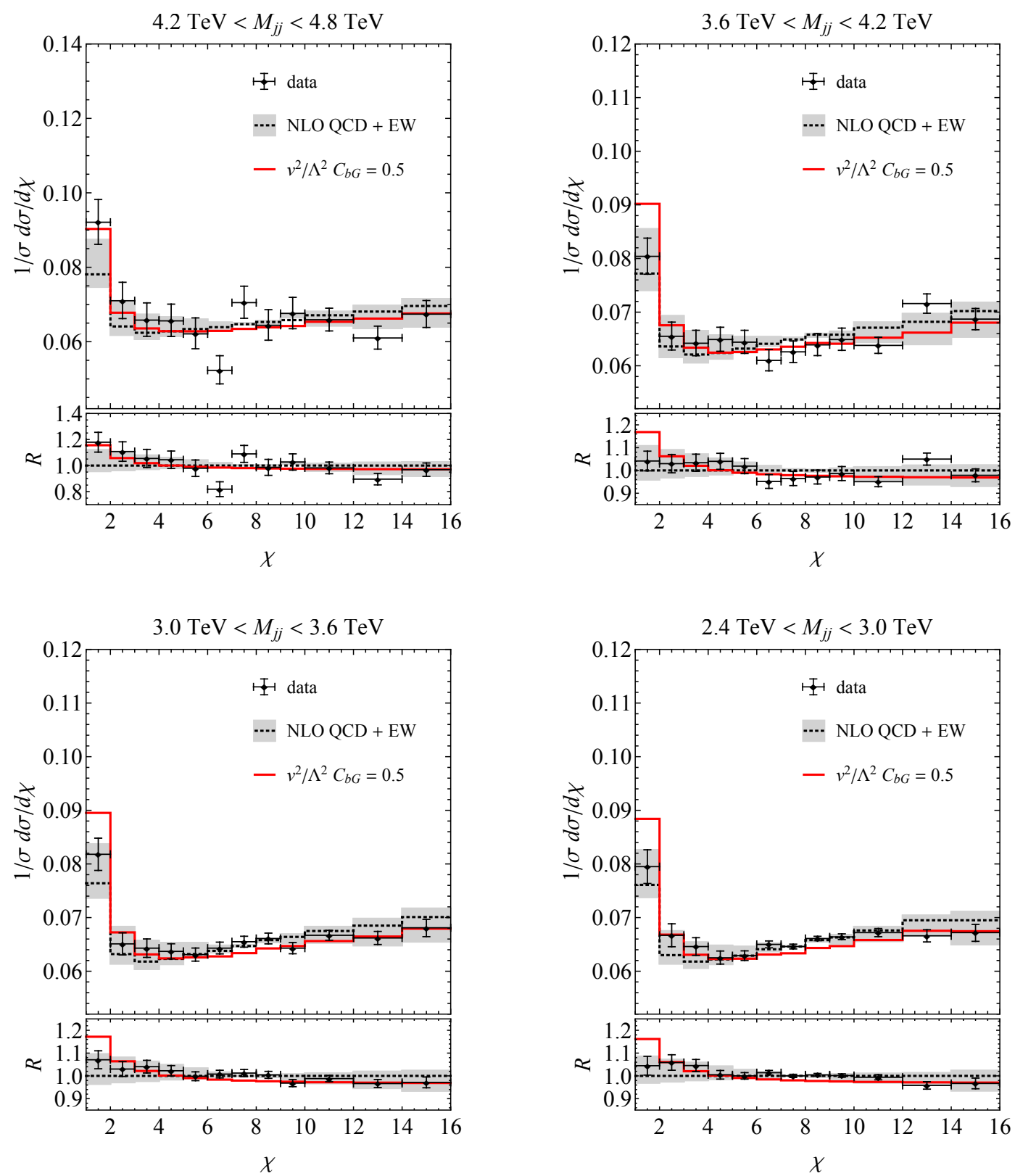

Figure 3. As figure 2 but for the four lower mass bins considered in the CMS analysis [19].

\section{Constraints from searches for $b$-jet pairs}

In addition to using data for unflavoured dijet production, one can also exploit the measurements of mass distributions of jet pairs with one or two jets identified as $b$-jets [20, 25-27] to constrain the Wilson coefficient of the operator $Q_{b G}$ - see also [12] for an earlier study.

To calculate the differential cross sections for the production of $b$-jet pairs we have used a FeynRules 2 [28] implementation of the operator $Q_{b G}$ in the UFO format [29]. The generation and showering of the samples has been performed at LO with MadGraph5_aMCNLO [30] and PYTHIA 8.2 [31], respectively, using NNPDF31_nlo_as_0118 PDFs [32] and working in the four-flavour scheme. The background distribution was corrected to the NLO prediction 
using the matrix elements calculated in [33] as implemented in MCFM [34]. Hadronic jets are built using the anti- $k_{t}$ algorithm [35] with a radius parameter of $R=0.4$, as implemented in Fast Jet [36]. We furthermore rely on DELPHES 3 [37] as a fast detector simulation and on CheckMATE 2 [38]. Our event selection follows the ATLAS analysis [20] which is based on $139 \mathrm{fb}^{-1}$ of dijet data collected at $13 \mathrm{TeV} \mathrm{CM}$ energy. We require at least two jets $(j)$ with a transverse momentum $p_{T}(j)$ satisfying $p_{T}(j)>150 \mathrm{GeV}$ and the azimuthal angle difference $\Delta \phi\left(j_{1} j_{2}\right)$ between the two leading jets $j_{1}$ and $j_{2}$ must fulfill $\left|\Delta \phi\left(j_{1} j_{2}\right)\right|>1.0$. The two leading jets must be $b$-tagged and their pseudorapidities must satisfy $|\eta(j)|<2.0$. The $b$-tagging algorithm is taken from the ATLAS publication [39], and in accordance with [20] a $b$-tagging working point is chosen that yields a $b$-tagging efficiency of $77 \%$, a $c$-jet rejection of 5 and a light-flavour jet rejection of 110. Furthermore, to suppress the QCD background a selection cut of $\left|y_{*}\right|<0.8$ is imposed, where $y_{*}=\left(y\left(j_{1}\right)-y\left(j_{2}\right)\right) / 2$ with $y\left(j_{1}\right)$ and $y\left(j_{2}\right)$ the rapidities of the leading and subleading jet, respectively. Both the QCD background and the new-physics samples are generated binned in $p_{T}(j)$ and the resulting dijet mass distributions are fit to the parametric function

$$
f(x)=p_{1}(1-x)^{p_{2}} x^{p_{3}+p_{4} \ln x},
$$

where $x=M_{j j} / \sqrt{s}$ and $p_{i}$ with $i=1,2,3,4$ the four fitting parameters. Given the datadriven background estimate, the ATLAS measurement [20] is not a measurement of the absolute cross section of dijet production, making it insensitive to small overall shifts in the $b \bar{b}$ production rates. The ATLAS analysis is however sensitive to the shape of the dijet mass distribution, which can be determined in normalised rates.

In figure 4 we show the results for the normalised invariant dijet mass spectra requiring two $b$-jets. The black dotted line in the left plot denotes the central values of the background fit performed by ATLAS in [20], while the red curve shows our new-physics prediction for $v^{2} / \Lambda^{2} C_{b G}=0.05$. The grey band indicates the uncertainties associated to the background prediction as provided in [20]. On the right-hand side the ratio of the data and the newphysics prediction to the central value of the background fit is displayed. One observes that the contributions associated to $Q_{b G}$ lead to an enhancement in the tail of the $M_{j j}$ distribution with respect to the background prediction.

By performing a $\Delta \chi^{2}$ fit to the normalised spectra over the whole range of invariant dijet masses covered in the ATLAS study [20], i.e. $1133 \mathrm{GeV}<M_{j j}<4595 \mathrm{GeV}$, we obtain the following $95 \%$ CL limit

$$
\frac{\left|C_{b G}\right|}{\Lambda^{2}}<\left(\frac{1}{1.6 \mathrm{TeV}}\right)^{2}
$$

This bound is by a factor of about 2.5 better than the estimate that has been given in [12] based on the CMS dijet measurement [25]. Let us remark that adding an additional uncertainty of $10 \%$ to the background prediction obtained in [20] would lead to a slightly weaker bound than (3.2) of approximately $1 /(1.5 \mathrm{TeV})^{2}$. Since the unnormalised two $b$-tagged $\mathrm{MC}$ distribution and the data are found to agree within around $10 \%$ in the mass range $1 \mathrm{TeV}<M_{j j}<5 \mathrm{TeV}[26]$, we believe that the latter bound is very robust. Notice finally that searches for jet pairs with one or two jets identified as $c$-jets could be used to set a bound on the Wilson coefficient $\left|C_{c G}\right|$ similar to (3.2). To our knowledge dijet searches that employ $c$-tagging have however not been performed at the LHC to date. 

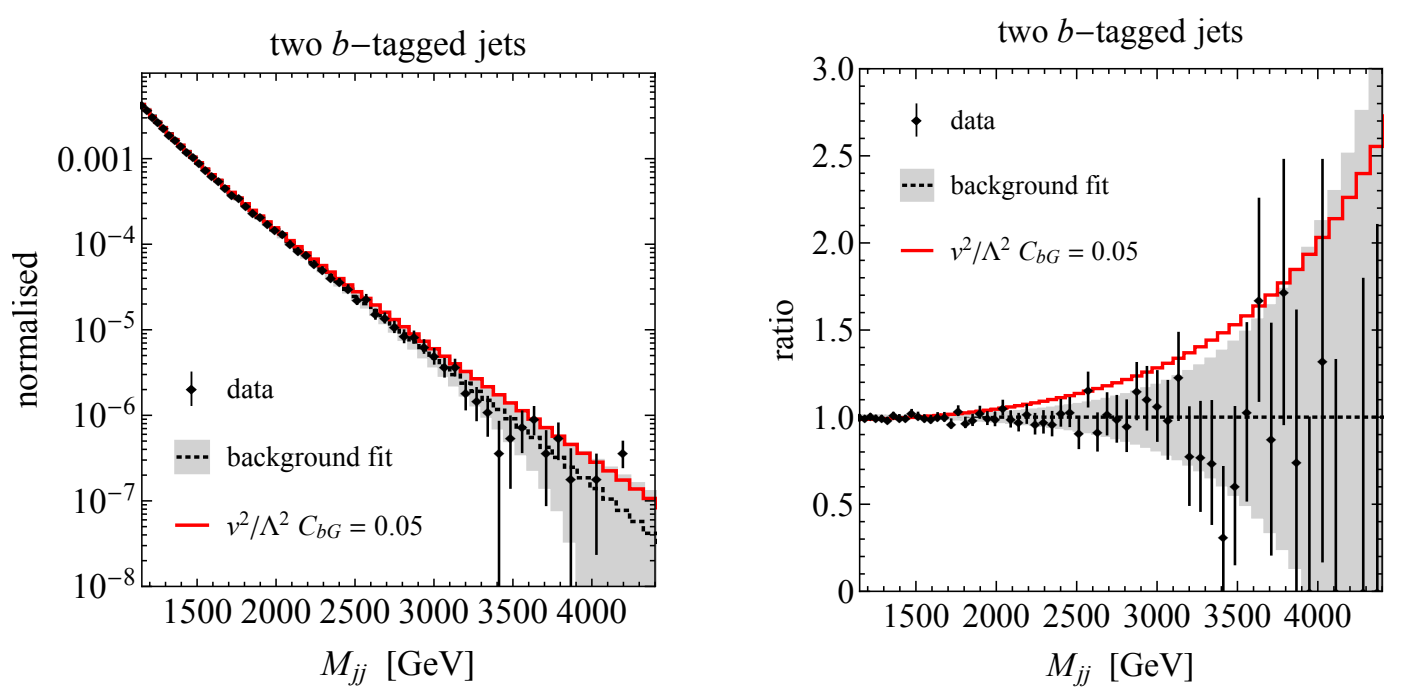

Figure 4. Left: normalised dijet invariant mass distributions for the category with two $b$-jets as measured by ATLAS in [20]. The black dotted line is the central value of the background fit performed by ATLAS, the grey band indicates the associated uncertainties and the red curve shows the new-physics prediction assuming $v^{2} / \Lambda^{2} C_{b G}=0.05$. Right: ratio of the data and the newphysics prediction for $v^{2} / \Lambda^{2} C_{b G}=0.05$ to the central value of the background fit. The colour coding resembles the one used in the left panel. Consult the main text for further details.

\section{Constraints from $Z$-boson production in association with $b$-jets}

Inclusive and differential measurements of $Z$-boson production in association with $b$-jets have been performed at the LHC by both the ATLAS and CMS collaborations [40-46]. These measurements provide not only an important role in improving our quantitative understanding of perturbative QCD (see e.g. [47]), but can also be exploited to search for BSM physics [48]. In this section we will consider the measurements performed in [45] to set bounds on the Wilson coefficient of the operator $Q_{b G}$.

We calculate the contribution of the operator $Q_{b G}$ to $p p \rightarrow b \bar{b} Z / \gamma^{*} \rightarrow b \bar{b} \ell^{+} \ell^{-}$at LO using MadGraph5_aMCNLO together with PYTHIA 8.2 and NNPDF31_nlo_as_0118 PDFs. The computation is performed in the four-flavour scheme. Relevant Feynman diagrams are shown in figure 5 . To be able compare our results to an existing analysis we employ the event selections of the recent ATLAS measurement [45] which is based on $35.6 \mathrm{fb}^{-1}$ of $13 \mathrm{TeV}$ LHC data. Electrons must satisfy the tight likelihood requirement [49], and are required to have a transverse momentum and pseudorapidity of $p_{T}(e)>27 \mathrm{GeV}$ and $|\eta(e)|<2.47$, respectively. Electron candidates in the transition region between the barrel and endcap electromagnetic calorimeters, i.e. $1.37<|\eta(e)|<1.52$, are excluded. Muons must satisfy medium identification criteria [50] and have to pass the requirements $p_{T}(\mu)>$ $27 \mathrm{GeV}$ and $|\eta(\mu)|<2.5$. Jets are reconstructed using the anti- $k_{t}$ algorithm with radius parameter $R=0.4$, and a $b$-tagging algorithm is applied with a $b$-tagging efficiency of $70 \%$ and misidentification rates of $8.3 \%$ of $0.26 \%$ for $c$-jets and a light-flavour jets, respectively. Events are required to have exactly two same-flavour $(\ell=e, \mu)$ opposite-sign leptons with a dilepton invariant mass in the range $76 \mathrm{GeV}<M_{\ell^{+} \ell^{-}}<106 \mathrm{GeV}$. To suppress the 

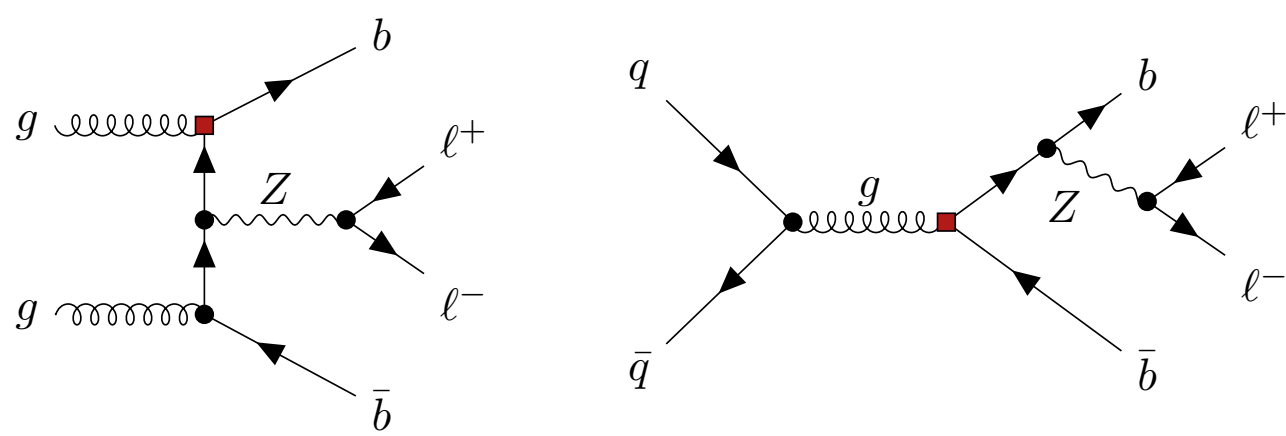

Figure 5. Examples of Feynman diagrams leading to a $b \bar{b} \ell^{+} \ell^{-}$signature at the LHC. The red squares denote the insertion of the bottom-quark chromomagnetic dipole moment. Graphs with a virtual photon $\gamma^{*}$ also contribute but are not shown explicitly.

background from $t \bar{t}$ events with dileptonic decays, events with $p_{T}\left(\ell^{+} \ell^{-}\right)<150 \mathrm{GeV}$ must also have missing transverse energy of $E_{T}^{\text {miss }}<60 \mathrm{GeV}$. Events passing the above selection are then assigned to two signal regions depending on the number of identified $b$-jets, and we concentrate in the following on the signal region with at least two $b$-jets. The object and event selection used in our analysis are implemented into CheckMATE 2 which uses DELPHES 3 as a fast detector simulation.

Several differential observables for the $p p \rightarrow b \bar{b} Z / \gamma^{*} \rightarrow b \bar{b} \ell^{+} \ell^{-}$process have been considered in the article [45]. Here we focus on the transverse momentum of the $Z$ boson $\left(p_{T}(Z)\right)$. In figure 6 we display results for the $p_{T}(Z)$ spectrum requiring two or more $b$-jets. The black dotted line and the grey band in the left panel are the SM prediction with its uncertainty, while the red curve shows our new-physics prediction assuming $v^{2} / \Lambda^{2} C_{b G}=0.07$. The used SM numbers correspond to those referred to as Sherpa 5FNS (NLO) in [45]. They have been obtained by ATLAS using the Sherpa v2 2.1 generator [51]. From the two panels it is evident that the relative effect of a non-zero contribution due to $Q_{b G}$ grows with transverse momentum and as a result the BSM prediction differs most visibly in the highest bin with $p_{T}(Z)>350 \mathrm{GeV}$.

Considering all seven bins of the ATLAS study [45] in a $\Delta \chi^{2}$ fit, we obtain

$$
\frac{\left|C_{b G}\right|}{\Lambda^{2}}<\left(\frac{1}{980 \mathrm{GeV}}\right)^{2}
$$

at $95 \%$ CL. We stress that this bound depends in a notable fashion on the systematic experimental and theoretical uncertainties that plague $p p \rightarrow b \bar{b} Z / \gamma^{*} \rightarrow b \bar{b} \ell^{+} \ell^{-}$at high $p_{T}(Z)$. In the bin with $p_{T}(Z)>350 \mathrm{GeV}$ the experimental (theoretical) uncertainty used in our analysis amounts to about $20 \%$ (35\%). Reducing the theoretical uncertainty by a factor of 2 would improve the limit (4.1) to approximately $1 /(1.1 \mathrm{TeV})^{2}$. In view of the recent progress [47] in the calculation of $Z$-boson production in association with $b$-jets at nextto-next-to-leading order accuracy in QCD including finite heavy-quark mass effects, such a reduction of uncertainties does not seem unreasonable. 

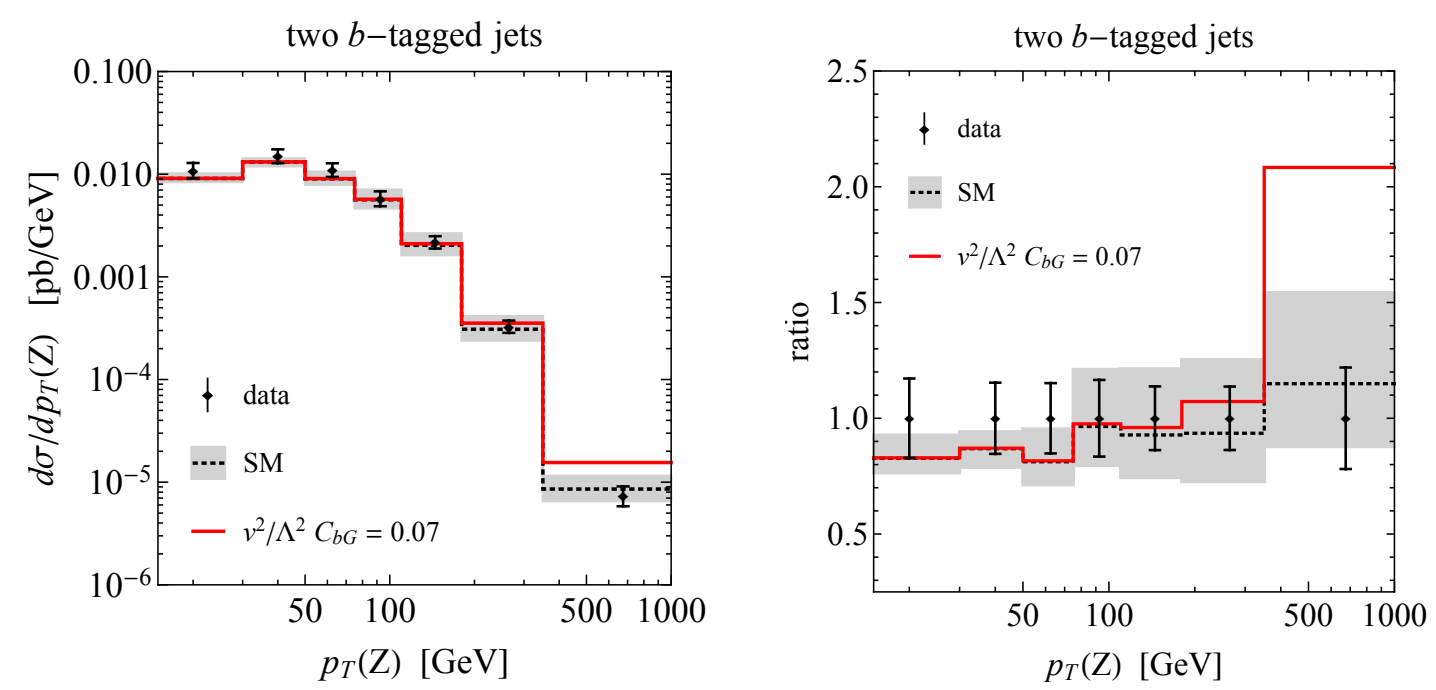

Figure 6. Left: $p_{T}(Z)$ distributions for the category with two $b$-jets. The black dotted line is the central value of the SM prediction provided by ATLAS [45], the grey band indicates the associated uncertainties and the red curve shows the new-physics prediction assuming $v^{2} / \Lambda^{2} C_{b G}=0.07$. Right: ratio between the SM and the BSM prediction for $v^{2} / \Lambda^{2} C_{b G}=0.07$ and the measurement. The colour coding resembles the one employed on the left-hand side.

\section{Constraints from flavour physics}

In order to derive a bound on the real part of the Wilson coefficients of the charm-quark dipole operator, let us consider the following effective interaction

$$
\mathcal{L}_{\text {eff }} \supset-\tilde{\mu}_{c}\left(m_{h}\right) \frac{g_{s}\left(m_{h}\right)}{2} \bar{c} \sigma_{\mu \nu} T^{a} c G^{a, \mu \nu},
$$

where the initial condition $\tilde{\mu}_{c}\left(m_{h}\right)$ of the charm-quark chromomagnetic dipole moment in the terms of the relevant Wilson coefficient multiplying the operators in (1.1) is given by

$$
\tilde{\mu}_{c}\left(m_{h}\right)=-\frac{\sqrt{2} v}{\Lambda^{2}} \operatorname{Re}\left(C_{c G}\right) .
$$

One-loop Feynman diagrams involving a $W$-boson exchange generate the chromomagnetic dipole operator

$$
Q_{8}=\frac{g_{s}}{(4 \pi)^{2}} m_{b} \bar{s}_{L} \sigma_{\mu \nu} T^{a} b_{R} G^{a, \mu \nu},
$$

which appears in the $\Delta B=1$ Lagrangian $\mathcal{L}=-4 G_{F} / \sqrt{2} V_{t s}^{*} V_{t b} C_{8} Q_{8}$ with $G_{F} \simeq 1 /\left(\sqrt{2} v^{2}\right)$ the Fermi constant and $V_{i j}$ the elements of the Cabibbo-Kobayashi-Maskawa (CKM) matrix. The corresponding matching correction reads $[52,53]$

$$
\delta C_{8}\left(m_{h}\right)=\frac{\bar{m}_{c}\left(m_{h}\right)}{2} \frac{V_{c s}^{*} V_{c b}}{V_{t s}^{*} V_{t b}} \tilde{\mu}_{c}\left(m_{h}\right),
$$

where $\bar{m}_{c}\left(m_{h}\right)$ denotes the charm-quark $\overline{\mathrm{MS}}$ mass evaluated at the Higgs-boson mass threshold. In terms of the shift $\delta C_{8}\left(m_{h}\right)$, the ratio between the branching ratio of $B \rightarrow X_{s} \gamma$ 
and its SM prediction can be written as [54]

$$
R_{X_{s}}=\frac{\operatorname{BR}\left(B \rightarrow X_{s} \gamma\right)}{\operatorname{BR}\left(B \rightarrow X_{s} \gamma\right)_{\mathrm{SM}}}=1-0.59 \delta C_{8}\left(m_{h}\right) .
$$

Combining the SM calculation of $B \rightarrow X_{s} \gamma$ with the present world average, one gets [55]

$$
R_{X_{s}}=0.97 \pm 0.08
$$

if uncertainties are added in quadrature. Using now that $V_{c s}^{*} V_{c b} \simeq-V_{t s}^{*} V_{t b}$ to high accuracy and employing $m_{c}\left(m_{h}\right) \simeq 0.87 \mathrm{GeV}$, one obtains from (5.2) to (5.6) the following 95\% CL limit

$$
\frac{\left|\operatorname{Re}\left(C_{c G}\right)\right|}{\Lambda^{2}}<\left(\frac{1}{25 \mathrm{GeV}}\right)^{2}
$$

We add that a bound that is weaker than (5.7) by a factor of around 4 is obtained by confronting the SM prediction $\mathrm{BR}\left(B \rightarrow X_{s} g\right)_{\mathrm{SM}} \simeq 5 \cdot 10^{-3}[56,57]$ with the corresponding experimental limit that reads $\mathrm{BR}\left(B \rightarrow X_{s} g\right) \lesssim 10 \%$ [58]. An even weaker bound follows from the isospin asymmetry in $B \rightarrow K^{*} \gamma$ [59-61]. The above estimate shows clearly that indirect probes of the chromodipole operators through flavour-physics observables cannot compete with the constraints arising from dijets. Notice that this is particularly true for the case of $Q_{b G}$ where a matching correction to $Q_{8}$ first arises at the two-loop level leading to a further suppression of (5.4) by a factor of the order of $(4 \pi)^{2}$. We finally remark that $\mathrm{CP}$ violation in the $\Delta C=1$ sector - for instance in the form of the difference $\Delta A_{\mathrm{CP}}$ between the two direct CP asymmetries in $D \rightarrow K^{+} K^{-}$and $D \rightarrow \pi^{+} \pi^{-}$- also does not provide relevant bounds on $\operatorname{Im}\left(C_{b G}\right)$ due to the strong CKM and loop suppression.

\section{Constraints from nEDM}

Searches for EDMs are known to place stringent constraints on any new-physics scenario with additional sources of CP violation (see [52, 53, 62-83] for reviews and recent discussions). To derive a bound on the imaginary part of the Wilson coefficient of the bottomquark dipole operator appearing in (1.1), we consider the following effective interactions

$$
\mathcal{L}_{\text {eff }} \supset-\tilde{d}_{b}\left(m_{h}\right) \frac{i g_{s}\left(m_{h}\right)}{2} \bar{b} \sigma_{\mu \nu} T^{a} \gamma_{5} b G^{a, \mu \nu}-w\left(m_{h}\right) \frac{1}{3} f^{a b c} G_{\mu \sigma}^{a} G_{\nu}^{b, \sigma} \widetilde{G}^{c, \mu \nu},
$$

where $\widetilde{G}^{a, \mu \nu}=1 / 2 \epsilon^{\mu \nu \alpha \beta} G_{\alpha \beta}^{a}$ is the dual field-strength tensor of QCD with $\epsilon^{\mu \nu \alpha \beta}$ the fully antisymmetric Levi-Civita tensor $\left(\epsilon^{0123}=1\right)$.

In the presence of the two dimension-six SMEFT operators (1.1) the initial condition $\tilde{d}_{b}\left(m_{h}\right)$ of the bottom-quark chromoelectric dipole moment is given by

$$
\tilde{d}_{b}\left(m_{h}\right)=-\frac{\sqrt{2} v}{\Lambda^{2}} \operatorname{Im}\left(C_{b G}\right),
$$

while the initial condition $w\left(m_{h}\right)$ of the Weinberg operator [84] vanishes. The bottomquark chromoelectric dipole moment has the following QCD running

$$
\tilde{d}_{b}\left(m_{b}\right)=\left(\frac{\alpha_{s}\left(m_{h}\right)}{\alpha_{s}\left(m_{b}\right)}\right)^{\frac{\gamma_{\tilde{d}}}{2 \gamma_{\alpha_{s}}}} \tilde{d}_{b}\left(m_{h}\right),
$$



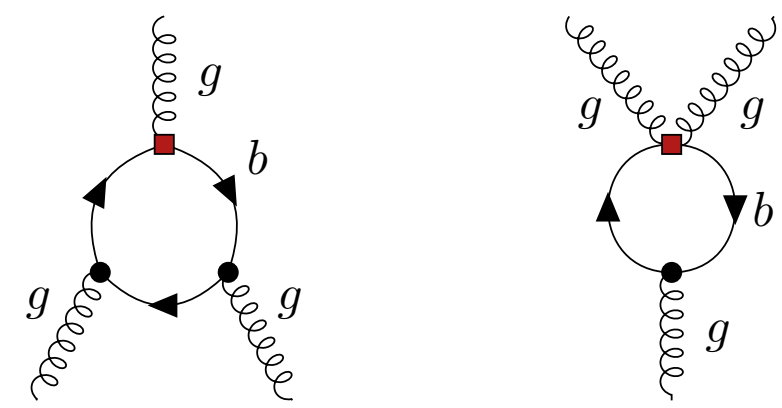

Figure 7. One-loop diagrams leading to a correction to the Weinberg operator at the bottom-quark threshold. The red squares denote the insertion of the bottom-quark chromoelectric dipole moment.

where $\gamma_{\alpha_{s}}$ denotes the LO anomalous dimension (or beta function) of the strong coupling constant and $\gamma_{\tilde{d}}$ is the LO anomalous dimension of the bottom-quark chromoelectric dipole moment. Explicitly, one has

$$
\gamma_{\alpha_{s}}=11-\frac{2}{3} N_{f}, \quad \gamma_{\tilde{d}}=\frac{4}{3},
$$

where the expression for $\gamma_{\alpha_{s}}$ can be found in any textbook on QCD and the result for $\gamma_{\tilde{d}}$ has first been presented in [85]. Notice that in (6.3) the number of active flavours is $N_{f}=5$ and that one could in principle resum large logarithms in the latter equation up to the next-to-next-to-leading logarithmic level because the self-mixing of the dipole operators is known up to the three-loop order [86]. Given the sizeable uncertainties of the hadronic matrix elements of the Weinberg operator and the chromoelectric dipole operators of light quarks (cf. the discussion before (6.8)) using only the leading-logarithmic (LL) result (6.3) is however fully justified.

At the bottom-quark mass threshold one integrates out the bottom quark. The corresponding Feynman diagrams are shown in figure 7. This gives a finite matching correction to the Weinberg operator [87-89]

$$
\delta w\left(m_{b}\right)=\frac{g_{s}^{3}\left(m_{b}\right)}{32 \pi^{2}} \frac{\tilde{d}_{b}\left(m_{b}\right)}{\bar{m}_{b}\left(m_{b}\right)} .
$$

Between the bottom-mass scale and the hadronic scale $\mu_{H} \simeq 1 \mathrm{GeV}$ the Weinberg operator mixes into itself and into the chromoelectric and electric dipole operators involving up and down quarks. As it turns out, the contributions from electric dipole operators are numerically very small and we thus neglect it in what follows. The LO anomalous dimensions describing the self-mixing of the Weinberg operator and the mixing of the Weinberg operator in the chromoelectric dipole operators, respectively, are [88, 90, 91]

$$
\gamma_{w}=3+2 N_{f}, \quad \gamma_{w \tilde{d}}=-6 .
$$

Resumming LL corrections in the four- and the three-flavour theory, we obtain

$$
w\left(\mu_{H}\right) \simeq 0.71 \delta w\left(m_{b}\right), \quad \tilde{d}_{q}\left(\mu_{H}\right) \simeq 0.14 \bar{m}_{q}\left(\mu_{H}\right) \delta w\left(m_{b}\right),
$$


where $q=u, d$. The numerical factors in (6.7) correspond to the following values $\alpha_{s}\left(m_{b}\right) \simeq 0.21, \alpha_{s}\left(m_{c}\right) \simeq 0.32$ and $\alpha_{s}\left(\mu_{H}\right) \simeq 0.36$ of the QCD coupling constant.

Estimates of the hadronic matrix elements of the Weinberg operator and the chromomagnetic dipole operators of the up and down quark are presently plagued by uncertainties of 50\% - see for instance [62, 92-98]. Adopting the QCD sum-rule estimates [92, 96] for the relevant hadronic matrix elements and employing $\bar{m}_{u}\left(\mu_{H}\right) \simeq 2.5 \cdot 10^{-3} \mathrm{GeV}$ and $\bar{m}_{d}\left(\mu_{H}\right) \simeq 5.4 \cdot 10^{-3} \mathrm{GeV}[99]$, the nEDM can be written as

$$
\frac{d_{n}}{e} \simeq(-1.77(1 \pm 0.5)+0.10(1 \pm 0.5)) \cdot 10^{-2} \mathrm{GeV} \delta w\left(m_{b}\right),
$$

where the first and the second term corresponds to the contribution from the Weinberg operator and the chromomagnetic dipole operators, respectively. Combining the above uncertainties in such a way that our prediction provides a lower absolute limit on the actual size of the $Q_{b G}$ correction to the nEDM, we find

$$
\left|\frac{d_{n}}{e}\right| \simeq 1.5 \cdot 10^{-22} \mathrm{~cm}\left(\frac{1 \mathrm{TeV}}{\Lambda}\right)^{2}\left|\operatorname{Im}\left(C_{b G}\right)\right| .
$$

Here we have employed $\alpha_{s}\left(m_{h}\right) \simeq 0.11$ and $\bar{m}_{b}\left(m_{b}\right) \simeq 4.2 \mathrm{GeV}$ [99] to obtain the numerical result. The current best experimental nEDM result [100] imposes the following $90 \%$ CL bound

$$
\left|\frac{d_{n}}{e}\right|<1.8 \cdot 10^{-26} \mathrm{~cm} .
$$

From (6.9) and (6.10) it follows that

$$
\frac{\left|\operatorname{Im}\left(C_{b G}\right)\right|}{\Lambda^{2}}<\left(\frac{1}{90 \mathrm{TeV}}\right)^{2}
$$

In the case of the charm-quark dipole operator entering (1.1), the derivation of the bound on the imaginary part of $C_{c G}$ follows the steps described above with minor modifications. The first modification is that from the initial scale $m_{h}$ the charm-quark chromomagnetic dipole moment $\tilde{d}_{c}$ runs to the charm-quark threshold $m_{c}$ in the five- and four-flavour theory. Second, the renormalisation group flow below $m_{c}$ where the Weinberg operator is generated - see $(6.5)$ - proceeds only in the three-flavour theory, leading to

$$
w\left(\mu_{H}\right) \simeq 0.94 \delta w\left(m_{c}\right), \quad \tilde{d}_{q}\left(\mu_{H}\right) \simeq 0.04 \bar{m}_{q}\left(\mu_{H}\right) \delta w\left(m_{c}\right) .
$$

Notice that given the smaller scale separation between $m_{c}$ and $\mu_{H}$ compared to $m_{b}$ and $\mu_{H}$ the renormalisation group effects in (6.12) are notably smaller than those in (6.7). Instead of (6.8) one then finds the following expression

$$
\frac{d_{n}}{e} \simeq(-2.34(1 \pm 0.5)+0.03(1 \pm 0.5)) \cdot 10^{-2} \mathrm{GeV} \delta w\left(m_{c}\right),
$$

which is fully dominated by the contribution from the Weinberg operator. This leads to

$$
\left|\frac{d_{n}}{e}\right| \simeq 1.4 \cdot 10^{-21} \mathrm{~cm}\left(\frac{1 \mathrm{TeV}}{\Lambda}\right)^{2}\left|\operatorname{Im}\left(C_{c G}\right)\right|,
$$

where we have used $\bar{m}_{c}\left(m_{c}\right) \simeq 1.3 \mathrm{GeV}$ [99]. Combining (6.10) and (6.14) we finally obtain

$$
\frac{\left|\operatorname{Im}\left(C_{c G}\right)\right|}{\Lambda^{2}}<\left(\frac{1}{275 \mathrm{TeV}}\right)^{2} .
$$




\begin{tabular}{|cccc|}
\hline Observable & Wilson coefficient & 95\% CL bound & Scale \\
\hline Dijet angular distributions & $\left|C_{b G}\right|,\left|C_{c G}\right|$ & 6.8 & $380 \mathrm{GeV}$ \\
Two $b$-tagged jets & $\left|C_{b G}\right|$ & 0.36 & $1.6 \mathrm{TeV}$ \\
$Z$-boson production with two b-jets & $\left|C_{b G}\right|$ & 1.04 & $980 \mathrm{GeV}$ \\
Inclusive radiative $B$ decay & $\left|\operatorname{Re}\left(C_{c G}\right)\right|$ & 1600 & $25 \mathrm{GeV}$ \\
Searches for nEDM & $\left|\operatorname{Im}\left(C_{b G}\right)\right|$ & $1.2 \cdot 10^{-4}$ & $90 \mathrm{TeV}$ \\
Searches for nEDM & $\left|\operatorname{Im}\left(C_{c G}\right)\right|$ & $1.3 \cdot 10^{-5}$ & $275 \mathrm{TeV}$ \\
\hline
\end{tabular}

Table 1. Summary of the constraints on the Wilson coefficients of the operators $Q_{b G}$ and $Q_{c G}$ derived in this article. The given $95 \% \mathrm{CL}$ bounds correspond to $\Lambda=1 \mathrm{TeV}$, while the numbers quoted for the suppression scale assume a purely real or imaginary Wilson coefficient with a magnitude of 1. See text for further explanations.

\section{Discussion}

In table 1 we summarise the limits on the Wilson coefficients of the operators $Q_{b G}$ and $Q_{c G}$ that we have derived in the course of this work. For what concerns the magnitudes of $C_{b G}$ and $C_{c G}$, one sees that searches for dijets final states provide bounds on the Wilson coefficients of order one assuming a suppression scale $\Lambda$ of $1 \mathrm{TeV}$. In fact, the nominal strongest limit derives at present from the ATLAS search for $b$-jet pairs performed in [20]. This search probes dijet invariant masses in the range $1133 \mathrm{GeV}<M_{j j}<4595 \mathrm{GeV}$, which pushes the Wilson coefficient to $\left|C_{b G}\right| \gtrsim 4 \pi$, if one requires $\Lambda \gtrsim 5 \mathrm{TeV}$ to cover the whole range of tested jet-jet invariant masses. Similar statements apply to the constraints following from the CMS measurement of the dijet angular distributions [19]. On general grounds, the limits (2.5) and (3.2) therefore only constrain strongly-coupled ultraviolet (UV) completions - for related discussions of the applicability of the SMEFT to dijet searches see [13-18]. The situation is better in the case of the bound on $C_{b G}$ that we have derived from the recent ATLAS measurement [45] of $p p \rightarrow b \bar{b} Z / \gamma^{*} \rightarrow b \bar{b} \ell^{+} \ell^{-}$, because the energy scales tested by this measurement are all below $1 \mathrm{TeV}$. Notice that $p p \rightarrow b \bar{b} Z / \gamma^{*} \rightarrow b \bar{b} \ell^{+} \ell^{-}$measurements are systematically limited at the LHC but the prospects of reducing the systematic uncertainties due to an improved theoretical understanding are quite good (cf. [47]). These two features taken together make $Z$-boson production in association with $b$-jets in our opinion a key process to search and to constrain the chromomagnetic bottom-quark dipole operator at upcoming LHC runs. Another interesting avenue to constrain chromomagnetic dipole operators involving the bottom quark is HL-LHC Higgs physics [11, 12], and we plan to return to this topic in future research. Furthermore, as can be learnt from table 1 as well as the discussion at the end of section 5 , flavour physics does not provide a meaningful bound on either the operator $Q_{b G}$ or the operator $Q_{c G}$. We finally add that HL-LHC constraints on $\left|C_{b G}\right|$ from Higgs physics have been derived in [11, 12]. For instance, the article [12] obtained $\left|C_{b G}\right| / \Lambda^{2}<1 /(2.1 \mathrm{TeV})^{2}$ by considering $p p \rightarrow b \bar{b} h \rightarrow b \bar{b} \gamma \gamma$ production.

From table 1 it is also clear that in contrast to $\left|C_{b G}\right|$ and $\left|C_{c G}\right|$, the imaginary parts of both Wilson coefficients are severely constrained by searches for a nEDM. In the case of a 
purely imaginary Wilson coefficient $C_{b G}\left(C_{c G}\right)$ of 1 , scales as high as $90 \mathrm{TeV}(275 \mathrm{TeV})$ are excluded at $90 \%$ CL by these indirect searches. Notice that in weakly-coupled UV complete theories the Wilson coefficients of chromodipole operators are typically both Yukawa- and loop-suppressed. Assuming that $C_{b G}=y_{b} /(4 \pi)^{2}$ and $C_{c G}=y_{c} /(4 \pi)^{2}$ the bounds (6.11) and (6.15) imply $\Lambda>960 \mathrm{GeV}$ and $\Lambda>1.5 \mathrm{TeV}$, respectively. This numerical example shows that nEDM searches, unlike the studied collider constraints, are able to test weaklycoupled TeV-scale new-physics models. Making effects of bottom-quark and charm-quark chromodipole operators observable at the LHC therefore generically requires a mechanism that suppresses new sources of CP-violation beyond the SM.

\section{Acknowledgments}

UH thanks Darren Scott, Marius Wiesemann and Giulia Zanderighi for triggering this work and Rhorry Gauld for helpful correspondence concerning $p p \rightarrow b \bar{b} Z / \gamma^{*} \rightarrow b \bar{b} \ell^{+} \ell^{-}$production. We are also grateful to Amando Hala for reminding us of [100] and to Roman Zwicky for his interest in our work. All Feynman diagrams shown in this article have been drawn with TikZ-Feynman [101].

Open Access. This article is distributed under the terms of the Creative Commons Attribution License (CC-BY 4.0), which permits any use, distribution and reproduction in any medium, provided the original author(s) and source are credited.

\section{References}

[1] ATLAS collaboration, Observation of $H \rightarrow b \bar{b}$ decays and $V H$ production with the ATLAS detector, Phys. Lett. B $\mathbf{7 8 6}$ (2018) 59 [arXiv:1808.08238] [INSPIRE].

[2] CMS collaboration, Observation of Higgs boson decay to bottom quarks, Phys. Rev. Lett. 121 (2018) 121801 [arXiv: 1808.08242] [INSPIRE].

[3] F. Bishara, U. Haisch, P.F. Monni and E. Re, Constraining Light-Quark Yukawa Couplings from Higgs Distributions, Phys. Rev. Lett. 118 (2017) 121801 [arXiv:1606.09253] [INSPIRE].

[4] Y. Soreq, H.X. Zhu and J. Zupan, Light quark Yukawa couplings from Higgs kinematics, JHEP 12 (2016) 045 [arXiv: 1606.09621] [INSPIRE].

[5] ATLAS collaboration, Search for the Decay of the Higgs Boson to Charm Quarks with the ATLAS Experiment, Phys. Rev. Lett. 120 (2018) 211802 [arXiv: 1802.04329] [INSPIRE].

[6] CMS collaboration, Measurement and interpretation of differential cross sections for Higgs boson production at $\sqrt{s}=13 \mathrm{TeV}$, Phys. Lett. B 792 (2019) 369 [arXiv:1812.06504] [INSPIRE].

[7] CMS collaboration, A search for the standard model Higgs boson decaying to charm quarks, JHEP 03 (2020) 131 [arXiv: 1912.01662] [INSPIRE].

[8] ATLAS collaboration, Measurements and interpretations of Higgs-boson fiducial cross sections in the diphoton decay channel using $139 \mathrm{fb}^{-1}$ of pp collision data at $\sqrt{\mathrm{s}}=13 \mathrm{TeV}$ with the ATLAS detector, Tech. Rep. ATLAS-CONF-2019-029, CERN, Geneva (2019). 
[9] ATLAS collaboration, Measurements of the Higgs boson inclusive and differential fiducial cross sections in the 4 l decay channel at $\sqrt{s}=13$ TeV, Eur. Phys. J. C 80 (2020) 942 [arXiv: 2004.03969] [INSPIRE].

[10] R. Gauld, B.D. Pecjak and D.J. Scott, $Q C D$ radiative corrections for $h \rightarrow b \bar{b}$ in the Standard Model Dimension-6 EFT, Phys. Rev. D 94 (2016) 074045 [arXiv: 1607.06354] [INSPIRE].

[11] A. Hayreter and G. Valencia, Constraints on anomalous color dipole operators from Higgs boson production at the LHC, Phys. Rev. D 88 (2013) 034033 [arXiv:1304.6976] [InSPIRE].

[12] J. Bramante, A. Delgado, L. Lehman and A. Martin, Boosted Higgses from chromomagnetic

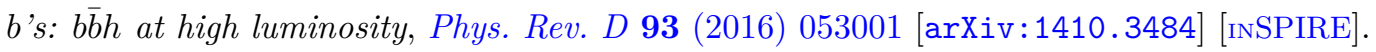

[13] F. Krauss, S. Kuttimalai and T. Plehn, LHC multijet events as a probe for anomalous dimension-six gluon interactions, Phys. Rev. D 95 (2017) 035024 [arXiv:1611.00767] [INSPIRE].

[14] S. Alioli, M. Farina, D. Pappadopulo and J.T. Ruderman, Precision Probes of QCD at High Energies, JHEP 07 (2017) 097 [arXiv: 1706. 03068] [INSPIRE].

[15] S. Alte, M. König and W. Shepherd, Consistent Searches for SMEFT Effects in Non-Resonant Dijet Events, JHEP 01 (2018) 094 [arXiv:1711.07484] [INSPIRE].

[16] V. Hirschi, F. Maltoni, I. Tsinikos and E. Vryonidou, Constraining anomalous gluon self-interactions at the LHC: a reappraisal, JHEP 07 (2018) 093 [arXiv: 1806. 04696] [INSPIRE].

[17] E. Keilmann and W. Shepherd, Dijets at Tevatron Cannot Constrain SMEFT Four-Quark Operators, JHEP 09 (2019) 086 [arXiv: 1907.13160] [INSPIRE].

[18] R. Goldouzian and M.D. Hildreth, LHC dijet angular distributions as a probe for the dimension-six triple gluon vertex, Phys. Lett. B 811 (2020) 135889 [arXiv:2001.02736] [INSPIRE].

[19] CMS collaboration, Search for new physics in dijet angular distributions using proton-proton collisions at $\sqrt{s}=13 \mathrm{TeV}$ and constraints on dark matter and other models, Eur. Phys. J. C 78 (2018) 789 [arXiv:1803.08030] [InSPIRE].

[20] ATLAS collaboration, Search for new resonances in mass distributions of jet pairs using $139 \mathrm{fb}^{-1}$ of pp collisions at $\sqrt{s}=13 \mathrm{TeV}$ with the ATLAS detector, JHEP 03 (2020) 145 [arXiv: 1910.08447] [INSPIRE].

[21] CMS collaboration, Search for high mass dijet resonances with a new background prediction method in proton-proton collisions at $\sqrt{s}=13 \mathrm{TeV}$, JHEP 05 (2020) 033

[arXiv: 1911.03947] [INSPIRE].

[22] ATLAS collaboration, Dijet resonance search with weak supervision using $\sqrt{s}=13 \mathrm{TeV} p p$ collisions in the ATLAS detector, Phys. Rev. Lett. 125 (2020) 131801 [arXiv:2005.02983] [INSPIRE].

[23] S. Dulat et al., New parton distribution functions from a global analysis of quantum chromodynamics, Phys. Rev. D 93 (2016) 033006 [arXiv:1506.07443] [InSPIRE].

[24] D.B. Clark, E. Godat and F.I. Olness, ManeParse: A Mathematica reader for Parton Distribution Functions, Comput. Phys. Commun. 216 (2017) 126 [arXiv:1605.08012] [INSPIRE].

[25] CMS collaboration, Search for Heavy Resonances Decaying into bb and bg Final States in $p p$ Collisions at sqrt(s) $=8 \mathrm{TeV}$, Tech. Rep. CMS-PAS-EXO-12-023, CERN, Geneva (2013). 
[26] ATLAS collaboration, Search for resonances in the mass distribution of jet pairs with one or two jets identified as b-jets in proton-proton collisions at $\sqrt{s}=13$ TeV with the ATLAS detector, Phys. Rev. D 98 (2018) 032016 [arXiv:1805.09299] [INSPIRE].

[27] CMS collaboration, Search for low-mass resonances decaying into bottom quark-antiquark pairs in proton-proton collisions at $\sqrt{s}=13 \mathrm{TeV}$, Phys. Rev. D 99 (2019) 012005 [arXiv: 1810.11822] [INSPIRE].

[28] A. Alloul, N.D. Christensen, C. Degrande, C. Duhr and B. Fuks, FeynRules 2.0 - A complete toolbox for tree-level phenomenology, Comput. Phys. Commun. 185 (2014) 2250 [arXiv: 1310.1921] [INSPIRE].

[29] C. Degrande, C. Duhr, B. Fuks, D. Grellscheid, O. Mattelaer and T. Reiter, UFO - The Universal FeynRules Output, Comput. Phys. Commun. 183 (2012) 1201 [arXiv:1108.2040] [INSPIRE].

[30] J. Alwall et al., The automated computation of tree-level and next-to-leading order differential cross sections, and their matching to parton shower simulations, JHEP 07 (2014) 079 [arXiv: 1405.0301] [INSPIRE].

[31] T. Sjöstrand et al., An introduction to PYTHIA 8.2, Comput. Phys. Commun. 191 (2015) 159 [arXiv:1410.3012] [INSPIRE].

[32] NNPDF collaboration, Parton distributions from high-precision collider data, Eur. Phys. J. C 77 (2017) 663 [arXiv:1706.00428] [INSPIRE].

[33] P. Nason, S. Dawson and R.K. Ellis, The Total Cross-Section for the Production of Heavy Quarks in Hadronic Collisions, Nucl. Phys. B 303 (1988) 607 [InSPIRE].

[34] J. Campbell and T. Neumann, Precision Phenomenology with MCFM, JHEP 12 (2019) 034 [arXiv: 1909.09117] [INSPIRE].

[35] M. Cacciari, G.P. Salam and G. Soyez, The anti-k $k_{t}$ jet clustering algorithm, JHEP 04 (2008) 063 [arXiv: 0802.1189] [INSPIRE].

[36] M. Cacciari, G.P. Salam and G. Soyez, FastJet User Manual, Eur. Phys. J. C 72 (2012) 1896 [arXiv:1111.6097] [INSPIRE].

[37] DELPHES 3 collaboration, DELPHES 3, A modular framework for fast simulation of a generic collider experiment, JHEP 02 (2014) 057 [arXiv:1307.6346] [INSPIRE].

[38] D. Dercks, N. Desai, J.S. Kim, K. Rolbiecki, J. Tattersall and T. Weber, CheckMATE 2: From the model to the limit, Comput. Phys. Commun. 221 (2017) 383 [arXiv:1611.09856] [INSPIRE].

[39] ATLAS collaboration, ATLAS b-jet identification performance and efficiency measurement with $t \bar{t}$ events in pp collisions at $\sqrt{s}=13 \mathrm{TeV}$, Eur. Phys. J. C 79 (2019) 970 [arXiv: 1907.05120] [INSPIRE].

[40] CMS collaboration, Measurement of the Cross Section and Angular Correlations for Associated Production of a $Z$ Boson with b Hadrons in pp Collisions at $\sqrt{s}=7 \mathrm{TeV}$, JHEP 12 (2013) 039 [arXiv:1310.1349] [INSPIRE].

[41] CMS collaboration, Measurement of the production cross sections for a $Z$ boson and one or more $b$ jets in pp collisions at $\sqrt{s}=7 \mathrm{TeV}$, JHEP 06 (2014) 120 [arXiv:1402.1521] [INSPIRE].

[42] ATLAS collaboration, Measurement of differential production cross-sections for a $Z$ boson in association with b-jets in $7 \mathrm{TeV}$ proton-proton collisions with the ATLAS detector, JHEP 10 (2014) 141 [arXiv: 1407.3643] [INSPIRE]. 
[43] CMS collaboration, Measurements of the associated production of a $Z$ boson and $b$ jets in pp collisions at $\sqrt{s}=8 \mathrm{TeV}$, Eur. Phys. J. C 77 (2017) 751 [arXiv:1611.06507] [INSPIRE].

[44] CMS collaboration, Measurement of the associated production of a $Z$ boson with charm or bottom quark jets in proton-proton collisions at $\sqrt{s}=13 \mathrm{TeV}$, Phys. Rev. D 102 (2020) 032007 [arXiv:2001.06899] [INSPIRE].

[45] ATLAS collaboration, Measurements of the production cross-section for a $Z$ boson in association with b-jets in proton-proton collisions at $\sqrt{s}=13$ TeV with the ATLAS detector, JHEP 07 (2020) 044 [arXiv: 2003.11960] [INSPIRE].

[46] ATLAS collaboration, Search for new phenomena in final states with two leptons and one or no b-tagged jets at $\sqrt{s}=13 \mathrm{TeV}$ using the ATLAS detector, Tech. Rep. ATLAS-CONF-2021-012, CERN, Geneva (2021).

[47] R. Gauld, A. Gehrmann-De Ridder, E.W.N. Glover, A. Huss and I. Majer, Predictions for $Z$-Boson Production in Association with a b-Jet at $\mathcal{O}\left(\alpha_{s}^{3}\right)$, Phys. Rev. Lett. 125 (2020) 222002 [arXiv:2005.03016] [INSPIRE].

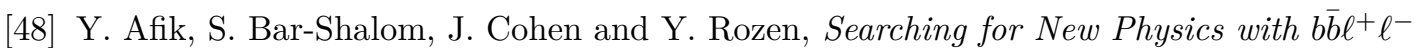
contact interactions, Phys. Lett. B 807 (2020) 135541 [arXiv:1912.00425] [InSPIRE].

[49] ATLAS collaboration, Electron reconstruction and identification in the ATLAS experiment using the 2015 and 2016 LHC proton-proton collision data at $\sqrt{s}=13$ TeV, Eur. Phys. J. C 79 (2019) 639 [arXiv: 1902.04655] [INSPIRE].

[50] ATLAS collaboration, Muon reconstruction performance of the ATLAS detector in proton-proton collision data at $\sqrt{s}=13 \mathrm{TeV}$, Eur. Phys. J. C 76 (2016) 292 [arXiv: 1603.05598] [INSPIRE].

[51] SHERPA collaboration, Event Generation with Sherpa 2.2, SciPost Phys. 7 (2019) 034 [arXiv: 1905.09127] [INSPIRE].

[52] F. Sala, A bound on the charm chromo-EDM and its implications, JHEP 03 (2014) 061 [arXiv:1312.2589] [INSPIRE].

[53] M. Gorbahn and U. Haisch, Searching for $t \rightarrow c(u) h$ with dipole moments, JHEP 06 (2014) 033 [arXiv: 1404.4873] [INSPIRE].

[54] M. Misiak et al., Updated NNLO QCD predictions for the weak radiative B-meson decays, Phys. Rev. Lett. 114 (2015) 221801 [arXiv:1503.01789] [INSPIRE].

[55] M. Misiak and M. Steinhauser, Weak radiative decays of the $B$ meson and bounds on $M_{H^{ \pm}}$ in the Two-Higgs-Doublet Model, Eur. Phys. J. C 77 (2017) 201 [arXiv:1702.04571] [INSPIRE].

[56] C. Greub and P. Liniger, The Rare decay $b \rightarrow s$ gluon beyond leading logarithms, Phys. Lett. $B 494$ (2000) 237 [hep-ph/0008071] [INSPIRE].

[57] C. Greub and P. Liniger, Calculation of next-to-leading QCD corrections to $b \rightarrow$ sg, Phys. Rev. D 63 (2001) 054025 [hep-ph/0009144] [INSPIRE].

[58] A. Kagan, The Phenomenology of enhanced $b \rightarrow s g$, in 2nd International Conference on $B$ Physics and CP-violation (BCONF 97) (1997) DOI [hep-ph/9806266] [InSPIRE].

[59] M. Dimou, J. Lyon and R. Zwicky, Exclusive Chromomagnetism in heavy-to-light FCNCs, Phys. Rev. D 87 (2013) 074008 [arXiv:1212.2242] [InSPIRE].

[60] J. Lyon and R. Zwicky, Isospin asymmetries in $B \rightarrow\left(K^{*}, \rho\right) \gamma / l^{+} l^{-}$and $B \rightarrow K l^{+} l^{-}$in and beyond the standard model, Phys. Rev. D 88 (2013) 094004 [arXiv:1305.4797] [INSPIRE]. 
[61] HFLAV collaboration, Averages of b-hadron, c-hadron, and $\tau$-lepton properties as of 2018, Eur. Phys. J. C 81 (2021) 226 [arXiv:1909.12524] [InSPIRE].

[62] M. Pospelov and A. Ritz, Electric dipole moments as probes of new physics, Annals Phys. 318 (2005) 119 [hep-ph/0504231] [inSPIRE].

[63] Y. Li, S. Profumo and M. Ramsey-Musolf, A Comprehensive Analysis of Electric Dipole Moment Constraints on CP-violating Phases in the MSSM, JHEP 08 (2010) 062 [arXiv: 1006.1440] [INSPIRE].

[64] J.F. Kamenik, M. Papucci and A. Weiler, Constraining the dipole moments of the top quark, Phys. Rev. D 85 (2012) 071501 [Erratum ibid. 88 (2013) 039903] [arXiv:1107.3143] [INSPIRE].

[65] D. McKeen, M. Pospelov and A. Ritz, Modified Higgs branching ratios versus CP and lepton flavor violation, Phys. Rev. D 86 (2012) 113004 [arXiv:1208.4597] [INSPIRE].

[66] W.-F. Chang, W.-P. Pan and F. Xu, Effective gauge-Higgs operators analysis of new physics associated with the Higgs boson, Phys. Rev. D 88 (2013) 033004 [arXiv:1303.7035] [INSPIRE].

[67] J. Engel, M.J. Ramsey-Musolf and U. van Kolck, Electric Dipole Moments of Nucleons, Nuclei, and Atoms: The Standard Model and Beyond, Prog. Part. Nucl. Phys. 71 (2013) 21 [arXiv: 1303.2371] [INSPIRE].

[68] M. Jung and A. Pich, Electric Dipole Moments in Two-Higgs-Doublet Models, JHEP 04 (2014) 076 [arXiv: 1308.6283] [INSPIRE].

[69] B. Gripaios and D. Sutherland, Searches for CP-violating dimension-6 electroweak gauge boson operators, Phys. Rev. D 89 (2014) 076004 [arXiv: 1309.7822] [INSPIRE].

[70] J. Brod, U. Haisch and J. Zupan, Constraints on CP-violating Higgs couplings to the third generation, JHEP 11 (2013) 180 [arXiv:1310.1385] [INSPIRE].

[71] S. Inoue, M.J. Ramsey-Musolf and Y. Zhang, CP-violating phenomenology of flavor conserving two Higgs doublet models, Phys. Rev. D 89 (2014) 115023 [arXiv:1403.4257] [INSPIRE].

[72] W. Altmannshofer, J. Brod and M. Schmaltz, Experimental constraints on the coupling of the Higgs boson to electrons, JHEP 05 (2015) 125 [arXiv:1503.04830] [INSPIRE].

[73] S. Dwivedi, D.K. Ghosh, B. Mukhopadhyaya and A. Shivaji, Constraints on CP-violating gauge-Higgs operators, Phys. Rev. D 92 (2015) 095015 [arXiv:1505.05844] [INSPIRE].

[74] Y.T. Chien, V. Cirigliano, W. Dekens, J. de Vries and E. Mereghetti, Direct and indirect constraints on CP-violating Higgs-quark and Higgs-gluon interactions, JHEP 02 (2016) 011 [arXiv:1510.00725] [INSPIRE].

[75] V. Cirigliano, W. Dekens, J. de Vries and E. Mereghetti, Is there room for CP-violation in the top-Higgs sector?, Phys. Rev. D 94 (2016) 016002 [arXiv: 1603.03049] [InSPIRE].

[76] V. Cirigliano, W. Dekens, J. de Vries and E. Mereghetti, Constraining the top-Higgs sector of the Standard Model Effective Field Theory, Phys. Rev. D 94 (2016) 034031 [arXiv: 1605. 04311] [INSPIRE].

[77] W. Dekens, J. de Vries, M. Jung and K.K. Vos, The phenomenology of electric dipole moments in models of scalar leptoquarks, JHEP 01 (2019) 069 [arXiv:1809.09114] [INSPIRE]. 
[78] C. Cesarotti, Q. Lu, Y. Nakai, A. Parikh and M. Reece, Interpreting the Electron EDM Constraint, JHEP 05 (2019) 059 [arXiv: 1810.07736] [INSPIRE].

[79] G. Panico, A. Pomarol and M. Riembau, EFT approach to the electron Electric Dipole Moment at the two-loop level, JHEP 04 (2019) 090 [arXiv:1810.09413] [INSPIRE].

[80] J. Brod and E. Stamou, Electric dipole moment constraints on CP-violating heavy-quark Yukawas at next-to-leading order, JHEP 07 (2021) 080 [arXiv:1810.12303] [INSPIRE].

[81] J. Brod and D. Skodras, Electric dipole moment constraints on CP-violating light-quark Yukawas, JHEP 01 (2019) 233 [arXiv:1811.05480] [INSPIRE].

[82] V. Cirigliano, A. Crivellin, W. Dekens, J. de Vries, M. Hoferichter and E. Mereghetti, $C P$ Violation in Higgs-Gauge Interactions: From Tabletop Experiments to the LHC, Phys. Rev. Lett. 123 (2019) 051801 [arXiv: 1903.03625] [INSPIRE].

[83] U. Haisch and A. Hala, Bounds on CP-violating Higgs-gluon interactions: the case of vanishing light-quark Yukawa couplings, JHEP 11 (2019) 117 [arXiv:1909.09373] [INSPIRE].

[84] S. Weinberg, Larger Higgs Exchange Terms in the Neutron Electric Dipole Moment, Phys. Rev. Lett. 63 (1989) 2333 [INSPIRE].

[85] M.A. Shifman, A.I. Vainshtein and V.I. Zakharov, On the Weak Radiative Decays (Effects of Strong Interactions at Short Distances), Phys. Rev. D 18 (1978) 2583 [Erratum ibid. 19 (1979) 2815] [INSPIRE].

[86] M. Gorbahn, U. Haisch and M. Misiak, Three-loop mixing of dipole operators, Phys. Rev. Lett. 95 (2005) 102004 [hep-ph/0504194] [INSPIRE].

[87] G. Boyd, A.K. Gupta, S.P. Trivedi and M.B. Wise, Effective Hamiltonian for the Electric Dipole Moment of the Neutron, Phys. Lett. B 241 (1990) 584 [InSPIRE].

[88] E. Braaten, C.-S. Li and T.-C. Yuan, The Evolution of Weinberg's Gluonic CP Violation Operator, Phys. Rev. Lett. 64 (1990) 1709 [INSPIRE].

[89] D. Chang, W.-Y. Keung, C.S. Li and T.C. Yuan, QCD Corrections to CP Violation From Color Electric Dipole Moment of b Quark, Phys. Lett. B 241 (1990) 589 [INSPIRE].

[90] E. Braaten, C.S. Li and T.C. Yuan, The Gluon Color - Electric Dipole Moment and Its Anomalous Dimension, Phys. Rev. D 42 (1990) 276 [INSPIRE].

[91] J. de Vries, G. Falcioni, F. Herzog and B. Ruijl, Two- and three-loop anomalous dimensions of Weinberg's dimension-six CP-odd gluonic operator, Phys. Rev. D 102 (2020) 016010 [arXiv: 1907.04923] [INSPIRE].

[92] M. Pospelov and A. Ritz, Neutron EDM from electric and chromoelectric dipole moments of quarks, Phys. Rev. D 63 (2001) 073015 [hep-ph/0010037] [INSPIRE].

[93] D.A. Demir, M. Pospelov and A. Ritz, Hadronic EDMs, the Weinberg operator, and light gluinos, Phys. Rev. D 67 (2003) 015007 [hep-ph/0208257] [INSPIRE].

[94] O. Lebedev, K.A. Olive, M. Pospelov and A. Ritz, Probing CP-violation with the deuteron electric dipole moment, Phys. Rev. D 70 (2004) 016003 [hep-ph/0402023] [INSPIRE].

[95] J. Hisano, J.Y. Lee, N. Nagata and Y. Shimizu, Reevaluation of Neutron Electric Dipole Moment with QCD Sum Rules, Phys. Rev. D 85 (2012) 114044 [arXiv:1204.2653] [INSPIRE].

[96] U. Haisch and A. Hala, Sum rules for CP-violating operators of Weinberg type, JHEP 11 (2019) 154 [arXiv: 1909.08955] [INSPIRE]. 
[97] N. Yamanaka and E. Hiyama, Weinberg operator contribution to the nucleon electric dipole moment in the quark model, Phys. Rev. D 103 (2021) 035023 [arXiv:2011.02531] [INSPIRE].

[98] Y. Hatta, Nucleon electric dipole moment from polarized deep inelastic scattering, Phys. Lett. B 814 (2021) 136126 [arXiv: 2012.01865] [INSPIRE].

[99] Particle Data Group collaboration, Review of Particle Physics, PTEP 2020 (2020) 083C01 [INSPIRE].

[100] NEDM collaboration, Measurement of the permanent electric dipole moment of the neutron, Phys. Rev. Lett. 124 (2020) 081803 [arXiv:2001.11966] [INSPIRE].

[101] J. Ellis, TikZ-Feynman: Feynman diagrams with TikZ, Comput. Phys. Commun. 210 (2017) 103 [arXiv: 1601.05437] [inSPIRE]. 